International Journal of Pure and Applied Mathematics

Volume 107 No. 3 2016, 615-624

ISSN: 1311-8080 (printed version); ISSN: 1314-3395 (on-line version)

url: http://www.ijpam.eu

doi: 10.12732/ijpam.v107i3.10

\title{
GRAPHICAL METHOD FOR INTERVAL BIMATRIX GAMES
}

\author{
Aykut Or ${ }^{1}$, G. Selin Savaşkan ${ }^{2}$, Yakup Haci ${ }^{3}$ \\ $1,2,3$ Department of Mathematics \\ Faculty of Arts and Sciences \\ Canakkale Onsekiz Mart University \\ Canakkale, TURKEY
}

\begin{abstract}
This paper deals with two-person non-zero sum games with interval payoffs. Graphical method to find a mixed strategy equilibrium is adapted to interval bimatrix games. In addition, interval bimatrix games Nash equilibrium is attained by graphical method. $\mathrm{Nu}-$ merical examples are also illustrated.
\end{abstract}

AMS Subject Classification: 91A05, 91A10

Key Words: interval bimatrix game, bimatrix game, interval payoff

\section{Introduction}

Interval game theory which is special case of fuzzy game theory, is an important content in interval fuzzy mathematics. Interval game has widely played an important role in the field of decision making theory such as economics, management, operation research etc. In this paper, we consider an interval based approach. Two-person non-zero sum games with interval payoff have already been studied in recent years by various researchers [1],[5],[6],[9]. Bimatrix game can be considered as a natural extension of the matrix game. Nash[2] defines the concept of Nash equilibrium solutions in bimatrix games for single pair of payoff matrices. Here, we present graphical method for solving interval bimatrix games by using

$$
\ell: \tilde{\mathbb{R}} \rightarrow \mathbb{R}, \quad \ell([a, b])=b-a .
$$

Received: November 5, 2015

Published: April 21, 2016

$\S$ Correspondence author (c) 2016 Academic Publications, Ltd. url: www.acadpubl.eu 


\section{Interval Numbers}

An interval number $\tilde{a}$ is closed subset of real numbers. Moreover its represented as follows,

$$
\tilde{a}=\left[a_{L}, a_{R}\right]=\left\{x \in \mathbb{R}: a_{L} \leq x \leq a_{R}\right\}
$$

in which $a_{L}$ and $a_{R}$ are respectively referred to as the lower and upper bound of the interval $\tilde{a}$ and $a_{L} \leq a_{R}$. If $a_{L}=a_{R}$, then $\tilde{a}=[a, a]$ is a real number. Midpoint and half-width of an interval number $\tilde{a}$ is defined as follows,

$$
m(\tilde{a})=\frac{a_{L}+a_{R}}{2}, w(\tilde{a})=\frac{a_{R}-a_{L}}{2} .
$$

The set of all interval numbers is represented by $\tilde{\mathbb{R}}$.

\subsection{Basic Interval Arithmetic}

Let $\tilde{a}=\left[a_{L}, a_{R}\right]$ and $\tilde{b}=\left[b_{L}, b_{R}\right]$ be two interval numbers. The arithmetic operations are defined as follows,

1. $\tilde{a}+\tilde{b}=\left[a_{L}+b_{L}, a_{R}+b_{R}\right]$;

2. $\tilde{a}-\tilde{b}=\left[a_{L}-b_{R}, a_{R}-b_{L}\right]$;

3. $\tilde{a} \tilde{b}=[\min S, \max S], S=\left\{a_{L} b_{L}, a_{L} b_{R}, a_{R} b_{L}, a_{R} b_{R}\right\}$;

4. $\frac{\tilde{a}}{b}=\tilde{a}(1 / \tilde{b}),\left(0 \notin \tilde{b}\right.$ and $\frac{1}{b}=\left\{\tilde{b}:\left(\frac{1}{b}\right) \in \tilde{b}\right\}=\left[\frac{1}{b_{R}}, \frac{1}{b_{L}}\right] \frac{\tilde{a}}{b}=\tilde{a}\left(\frac{1}{b}\right)=$ $\left.\left[a_{L}, a_{R}\right]\left[\frac{1}{b_{R}}, \frac{1}{b_{L}}\right]=\left[\min \left\{\frac{a_{L}}{b_{R}}, \frac{a_{L}}{b_{L}}, \frac{a_{R}}{b_{R}}, \frac{a_{R}}{b_{L}}\right\}, \max \left\{\frac{a_{L}}{b_{R}}, \frac{a_{L}}{b_{L}}, \frac{a_{R}}{b_{R}}, \frac{a_{R}}{b_{L}}\right\}\right]\right) ;$

5. $\alpha \in \mathbb{R}$ için $\alpha a=\alpha\left[a_{L}, a_{R}\right]=\left\{. \alpha\left[a_{L}, a_{R}\right], \quad \alpha \geq 0 \alpha\left[a_{R}, a_{L}\right], \quad \alpha<0\right.$;

6. $\tilde{a} \frac{1}{\tilde{a}} \neq 1$;

7. $\tilde{a}+(-\tilde{a}) \neq 0$.

\subsection{Comparasion of Interval Numbers}

An extensive research and wide coverage on interval arithmetic and its applications can be found in [4]. A brief comparasion on different interval orders is given in [4] on the basis of decision makers opinion.

Let $\tilde{a}=\left[a_{L}, a_{R}\right]$ and $\tilde{b}=\left[b_{L}, b_{R}\right]$ be two disjoint interval numbers. $\tilde{a}$ is less than $\tilde{b}$ if and only if $a_{R}<a_{L}$. This is denoted by $\tilde{a}<\tilde{b}$ and the relation 
is an extension of " $<$ " on the real line. If the closed interval numbers are overlapping, then we use the acceptability index idea suggested by [11].

Let $\tilde{\mathbb{R}}$ be the set of all closed intervals on the real line $\mathbb{R}$. The function

$$
\mathcal{A}: \tilde{\mathbb{R}} x \tilde{\mathbb{R}} \longrightarrow[\infty, 0)
$$

such that

$$
\mathcal{A}(\tilde{a} \prec \tilde{b})=\frac{m(\tilde{b})-m(\tilde{a})}{w(\tilde{b})+w(\tilde{a})}
$$

$(w(\tilde{b})+w(\tilde{a}) \neq 0)$ is called acceptability function. Thus, the number $\mathcal{A}(\tilde{a} \prec \tilde{b})$ is called grade of acceptability of the $\tilde{a}$ to be inferior to $\tilde{b}$.

1. $\mathcal{A}(\tilde{a} \prec \tilde{b}) \geq 1$ when $m(\tilde{b})>m(\tilde{a})$ and $a_{R} \leq b_{L}$

2. $0<\mathcal{A}(\tilde{a} \prec \tilde{b})<1$ when $m(\tilde{b})>m(\tilde{a})$ and $b_{L} \leq a_{R}$

3. $\mathcal{A}(\tilde{a} \prec \tilde{b})=0$ when $m(\tilde{b})=m(\tilde{a})$. In this case, an extensive research about comparasion of $\tilde{a}$ and $\tilde{b}$ can be found in [6].

Example1. Let $\tilde{a}=[10,20]$ and $\tilde{b}=[24,28]$ be two interval numbers.Then,

$$
\mathcal{A}(\tilde{a} \prec \tilde{b})=\frac{26-15}{2+5}=\frac{11}{7}>1 .
$$

Hence, $\tilde{a}$ is less than $\tilde{b}$ with full satisfaction.

Example 2. Let $\tilde{a}=[1,5]$ and $\tilde{b}=[3,17]$ be two interval numbers.Then,

$$
\mathcal{A}(\tilde{a} \prec \tilde{b})=\frac{10-3}{7+2}=\frac{7}{9} \in(0,1) .
$$

Hence, $\tilde{a}$ is less than $\tilde{b}$ with grade of satisfaction $\frac{7}{9}$.

\section{Interval Bimatrix Games}

A bimatrix game is a two player game, player $I$ and player $I I$, player $I$ has $m$ pure strategies $\left\{I_{1}, I_{2}, \ldots, I_{m}\right\}$ while player $I I$ has $n$ pure strategies $\left\{I I_{1}, I I_{2}, \ldots, I I_{n}\right\}$. There is no longer a value $c$, such that

$$
H_{I}\left(I_{i}, I I_{j}\right)+H_{I I}\left(I_{i}, I I_{j}\right)=c
$$


where $H_{I}\left(I_{i}, I I_{j}\right), H_{I I}\left(I_{i}, I I_{j}\right)$ are expected payoffs of player $I$ and player $I I$, respectively. That is, the pay-offs of player $I$ or player $I I$ do not give information about pay-off of the other one. Thus, if player $I$ plays $I_{\dot{I}}$ and player $I I$ plays $I I_{J}$, then the payoff is as follows

$$
H\left(I_{i}, I I_{j}\right)=\left(H_{I}\left(I_{i}, I I_{j}\right)+H_{I I}\left(I_{i}, I I_{j}\right)\right)
$$

If player $I$ selects the strategy $i$ and player $I I$ selects the strategy $j$, then $\left[a_{i j L}, a_{i j R}\right]$ and $\left[b_{i j L}, b_{i j R}\right]$ are payoffs of the player $I$ and player $I I$, respectively. Hence, a bimatrix game is determined by a pair of matrix $(\tilde{A}, \tilde{B})$. When payoff matrix whose entries are interval numbers, is given, the payyoff matris of twoperson non-zero sum game $(\tilde{A}, \tilde{B})$ is represented as follows:

$\left[\begin{array}{cccc}\left(\left[a_{11 L}, a_{11 R}\right],\left[b_{11 L}, b_{11 R}\right]\right) & \left(\left[a_{12 L}, a_{12 R}\right],\left[b_{12 L}, b_{12 R}\right]\right) & \ldots & \left(\left[a_{1 m L}, a_{1 m R}\right],\left[b_{1 m L}, b_{1 m R}\right]\right) \\ \left(\left[a_{21 L}, a_{21 R}\right],\left[b_{21 L}, b_{21 R}\right]\right) & \left(\left[a_{22 L}, a_{22 R}\right],\left[b_{22 L}, b_{22 R}\right]\right) & \cdots & \left(\left[a_{2 m L}, a_{2 m R}\right],\left[b_{2 m L}, b_{2 m R}\right]\right) \\ \vdots & \vdots & \vdots & \vdots \\ \left(\left[a_{1 m L}, a_{1 m R}\right],\left[b_{1 m L}, b_{1 m R}\right]\right) & \left(\left[a_{2 m L}, a_{2 m R}\right],\left[b_{2 m L}, b_{2 m R}\right]\right) & \cdots & \left(\left[a_{n m L}, a_{n m R}\right],\left[b_{n m L}, b_{n m R}\right]\right)\end{array}\right]$.

A mixed strategy set of player $I$ is

$$
S_{I}=\left\{x=\left(x_{1}, x_{2}, \ldots, x_{m}\right) \in \mathbb{R}^{m}: x_{i} \geq 0, \sum_{i=1}^{m} x_{i}=1 \forall i=1, \ldots, m\right\}
$$

Similarly, a mixed strategy set of player $I I$ is

$$
S_{I I}=\left\{y=\left(y_{1}, y_{2}, \ldots, y_{n}\right) \in \mathbb{R}^{n}: y_{i} \geq 0, \sum_{j=1}^{n} y_{i}=1 \forall i=1, \ldots, n\right\}
$$

When player $I$ plays mixed strategy of $x \in S_{I}$ and player $I I$ plays mixed strategy of $y \in S_{I I}$, player $I$ receives payoff

$$
H_{I}(x, y)=\sum_{j=1}^{n} \sum_{i=1}^{m} x_{i}\left[a_{i j L}, a_{i j R}\right] y_{j}
$$

and player $I I$ receives payoff

$$
H_{I I}(x, y)=\sum_{j=1}^{n} \sum_{i=1}^{m} x_{i}\left[b_{i j L}, b_{i j R}\right] y_{j} .
$$

Let $x^{*} \in S_{I}, y^{*} \in S_{I I}$ be mixed strategies for player $I$ and player $I I$, respectively. If the following inequalities

$$
H_{I}\left(x, y^{*}\right) \leq H_{I}\left(x^{*}, y^{*}\right)
$$




$$
H_{I I}\left(x^{*}, y\right) \leq H_{I I}\left(x^{*}, y^{*}\right)
$$

are satisfied for arbitrary $x \in S_{I}, y \in S_{I I}$ then pair of $\left(x^{*}, y^{*}\right)$ is called strategies of equilibrium (or pair of equilibrium) of the game.

\section{Graphical Method for Interval Bimatrix Games}

In a bimatrix game, we assume that the payoff matrixs of players whose strategies are not dominant. Let each of players have two strategy. The pay-off matrixs for player $I$ and player $I I$ are respectively as follows

$$
\begin{aligned}
&(\tilde{A}, \tilde{B})=\left(\begin{array}{ll}
\left(\left[a_{11 L}, a_{11 R}\right],\left[b_{11 L}, b_{11 R}\right]\right) & \left(\left[a_{12 L}, a_{12 R}\right],\left[b_{12 L}, b_{12 R}\right]\right) \\
\left(\left[a_{21 L}, a_{21 R}\right],\left[b_{21 L}, b_{21 R}\right]\right) & \left(\left[a_{22 L}, a_{22 R}\right],\left[b_{22 L}, b_{22 R}\right]\right)
\end{array}\right), \\
& \tilde{A}=\left(\begin{array}{ll}
{\left[a_{11 L}, a_{11 R}\right]} & {\left[a_{12 L}, a_{12 R}\right]} \\
{\left[a_{21 L}, a_{21 R}\right]} & {\left[a_{22 L}, a_{22 R}\right]}
\end{array}\right)=\left(\begin{array}{cc}
\tilde{a}_{11} & \tilde{a}_{12} \\
\tilde{a}_{21} & \tilde{a}_{22}
\end{array}\right), \\
& \tilde{B}=\left(\begin{array}{ll}
{\left[b_{11 L}, b_{11 R}\right]} & {\left[b_{12 L}, b_{12 R}\right]} \\
{\left[b_{21 L}, b_{21 R}\right]} & {\left[b_{22 L}, b_{22 R}\right]}
\end{array}\right)=\left(\begin{array}{ll}
\tilde{b}_{11} & \tilde{b}_{12} \\
\tilde{b}_{21} & \tilde{b}_{22}
\end{array}\right), \\
& h_{I}(x, y)= x^{T} \tilde{A}_{y} \\
&= x y\left(\tilde{a}_{11}+\tilde{a}_{22}-\tilde{a}_{21}-\tilde{a}_{12}\right)+x\left(\tilde{a}_{12}-\tilde{a}_{22}\right)+y\left(\tilde{a}_{21}-\tilde{a}_{22}\right)+\tilde{a}_{22}, \\
& h_{I I}(x, y)= x^{T} \widetilde{B}_{y} \quad x y\left(\tilde{b}_{11}+\tilde{b}_{22}-\tilde{b}_{21}-\tilde{b}_{12}\right)+x\left(\tilde{b}_{12}-\tilde{b}_{22}\right)+y\left(\tilde{b}_{21}-\tilde{b}_{22}\right)+\tilde{b}_{22}, \\
&= \tilde{A}_{y} \leq h_{I}(x, y)\left(\begin{array}{l}
{[1,1]} \\
{[1,1]}
\end{array}\right) .
\end{aligned}
$$

If (3) is edited, then we obtain (4) as follows

$$
\left.\begin{array}{l}
\tilde{a}_{11} y+\tilde{a}_{12}(1-y) \leq h_{I} \\
\tilde{a}_{21} y+\tilde{a}_{22}(1-y) \leq h_{I}
\end{array}\right\}
$$

If (1) is written in (3), then (5) is obtained as follows

$$
\left.\begin{array}{c}
\left(\tilde{a}_{11}+\tilde{a}_{22}-\tilde{a}_{21}-\tilde{a}_{12}\right)(1-x) y+\left(\tilde{a}_{12}-\tilde{a}_{22}\right)(1-x) \leq 0 \\
\left(\tilde{a}_{11}+\tilde{a}_{22}-\tilde{a}_{21}-\tilde{a}_{12}\right) x y+\left(\tilde{a}_{12}-\tilde{a}_{22}\right) x \geq 0
\end{array}\right\}
$$

Let $\tilde{p}$ and $\tilde{q}$ be as follows

$$
\tilde{p}=\tilde{a}_{11}+\tilde{a}_{22}-\tilde{a}_{21}-\tilde{a}_{12}
$$




$$
\tilde{q}=\tilde{a}_{12}-\tilde{a}_{22} .
$$

Moreover, we consider the length of interval $I(=[a, b])$ as $\ell(I)=b-a$. Let $\widetilde{\mathbb{R}}$ be set of all closed interval;

$$
\ell: \widetilde{\mathbb{R}} \longrightarrow \mathbb{R}, \quad \ell([a, b])=b-a
$$

The length is sample of set function. Therefore, domain of $I$ is set of interval and range of $I$ is also set of generalized real numbers. If we consider the above change of variable and the function $\ell$, then (5) can be written as follows,

$$
\left.\begin{array}{c}
\ell(\tilde{p})(1-x) y-\ell(\tilde{q})(1-x) \leq 0 \\
\ell(\tilde{p}) x y-\ell(\tilde{q}) x \geq 0
\end{array}\right\}
$$

Let's investigate the following conditions to determine $\mathrm{x}$ according to definition of mixed strategy:

(i) $x=0 \Longrightarrow \ell(\tilde{p}) y-\ell(\tilde{q}) \leq 0$;

(ii) $x=1 \Longrightarrow \ell(\tilde{p}) y-\ell(\tilde{q}) \geq 0$;

(iii) $0<x<1 \Longrightarrow \ell(\tilde{p}) y-\ell(\tilde{q})=0$.

Let $\mathrm{K}$ be solution set of (6), so $\mathrm{K}$ consists of the following conditions,

$$
\left.\begin{array}{ccc}
(0, y), & \ell(\tilde{p}) y-\ell(\tilde{q}) \leq 0 & y \in[0,1] \\
(1, y), & \ell(\tilde{p}) y-\ell(\tilde{q}) \geq 0 & y \in[0,1] \\
(x, y), & \ell(\tilde{p}) y-\ell(\tilde{q}) \geq 0 & y \in[0,1], x \in(0,1)
\end{array}\right\}
$$

If $\ell(\tilde{p})=\ell(\tilde{q})=0$, then (7) is satisfied for all $x$ and $y$. Therefore, the solution is all points $x \in[0,1], y \in[0,1]$ of unit square. If $\ell(\tilde{p})=0, \ell(\tilde{q}) \neq 0$, then $x=0$ or $x=1$. If $\ell(\tilde{p})>0$, then the solutions from (7) are obtained as follows,

$$
\left.\begin{array}{cc}
(0, y) & y \leq \alpha=\frac{\ell(\tilde{p})}{\ell(\tilde{q})} \\
(1, y) & y \geq \alpha \\
(x, y) & x \in(0,1), y=\alpha
\end{array}\right\}
$$

Hence, the curve of solutions set $\mathrm{K}$ for player $I$ can be represented in the plain:

Similarly, for player $I I$ :

$$
\tilde{B} x \leq h_{I I}(x, y)\left(\begin{array}{l}
{[1,1]} \\
{[1,1]}
\end{array}\right) .
$$




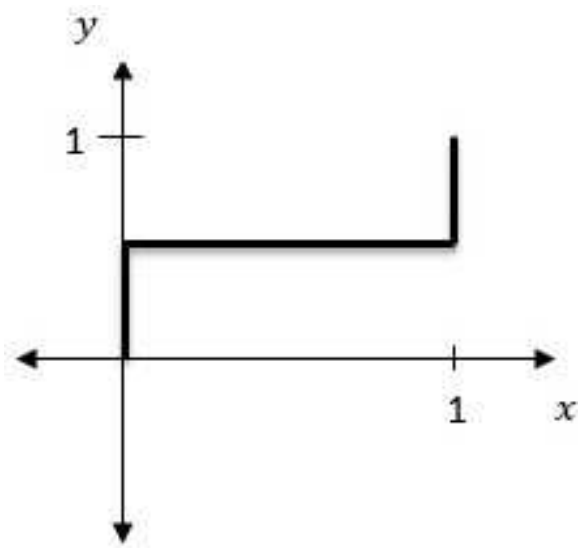

Figure 1

If value of $h_{I I}(x, y)$ is written in (8), then (9) is obtained as follows,

$$
\begin{aligned}
\left(\tilde{b}_{11}+\tilde{b}_{22}-\tilde{b}_{21}-\tilde{b}_{12}\right)(1-x) y+\left(\tilde{b}_{12}-\tilde{b}_{22}\right)(1-x) & \leq 0 \\
\left(\tilde{b}_{11}+\tilde{b}_{22}-\tilde{b}_{21}-\tilde{b}_{12}\right) x y+\left(\tilde{b}_{12}-\tilde{b}_{22}\right) x & \geq 0
\end{aligned}
$$

Let $\tilde{p}_{1}$ and $\tilde{q}_{1}$ be as follows,

$$
\begin{aligned}
\tilde{p}_{1} & =\tilde{b}_{11}+\tilde{b}_{22}-\tilde{b}_{21}-\tilde{b}_{12} \\
\tilde{q}_{1} & =\tilde{b}_{22}-\tilde{b}_{21}
\end{aligned}
$$

If we consider the above change of variable and the function $\ell$, then solution set $L$ for player $I I$ consists of the following conditions,

$$
\left.\begin{array}{ccc}
(x, 0), & \ell\left(\tilde{p}_{1}\right) y-\ell\left(\tilde{q}_{1}\right) \leq 0 & x \in[0,1] \\
(x, 1), & \ell\left(\tilde{p}_{1}\right) y-\ell\left(\tilde{q}_{1}\right) \geq 0 & x \in[0,1] \\
(x, y), & \ell\left(\tilde{p}_{1}\right) y-\ell\left(\tilde{q}_{1}\right)=0 & x \in[0,1], y \in(0,1)
\end{array}\right\}
$$

If $\ell\left(\tilde{p}_{1}\right)=\ell\left(\tilde{q}_{1}\right)=0$, then (10) is satisfied for all $x$ and $y$. Therefore, the solution is all points $x \in[0,1], y \in[0,1]$ of unit square. If $\ell\left(\tilde{p}_{1}\right)=0, \ell\left(\tilde{q}_{1}\right) \neq 0$, then $y=0$ or $y=1$. If $\ell\left(\tilde{p}_{1}\right)>0$, then the solutions from (10) are obtained as follows,

$$
\left.\begin{array}{cc}
(x, 0), & x \leq \beta=\frac{\ell\left(\tilde{q}_{1}\right)}{\ell\left(\tilde{p}_{1}\right)} \\
(x, 1), & x \geq \beta \\
(x, y), & y \in(0,1), x=\beta
\end{array}\right\}
$$

Hence, the curve of solutions set L for player $I I$ can be represented in the plain: 


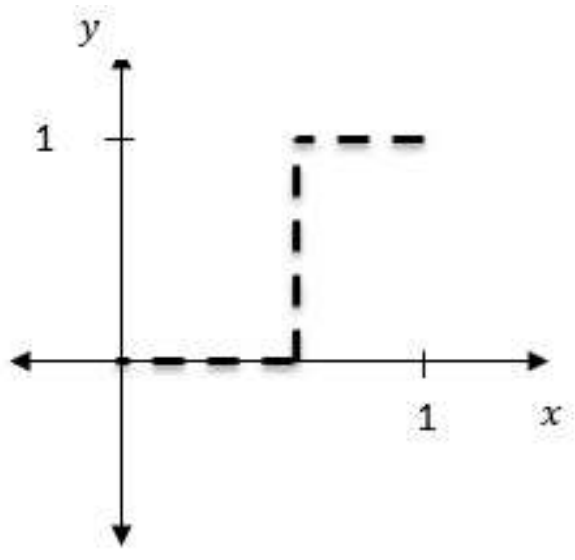

Figure 2

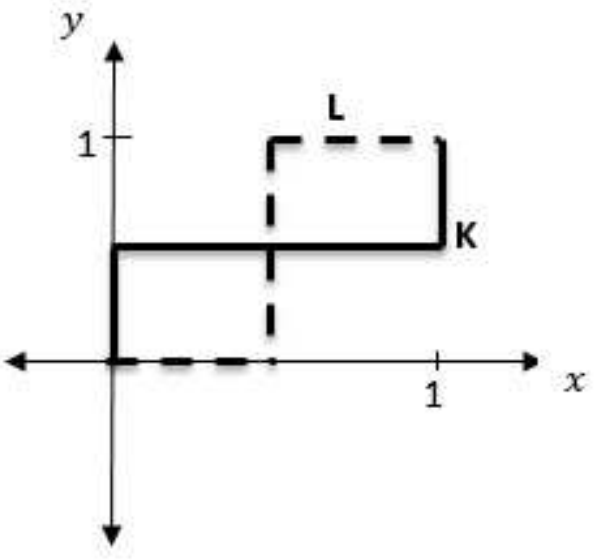

Figure 3

Solution of the game is $x$ and $y$ which are intersection of sets $K$ and $L$. Thus, the curve of bimatrix game can be represented in the plain:

Example. Let pay-off matrix be given as follows in a bimatrix game, determine game-value and equilibrium strategies

$$
(\tilde{A}, \tilde{B})=\left(\begin{array}{cc}
([1,3],[2,11]) & ([-2,12],[-1,3]) \\
([-5,8],[2,5]) & ([3,14],[1,6])
\end{array}\right) .
$$

\section{Solution.}




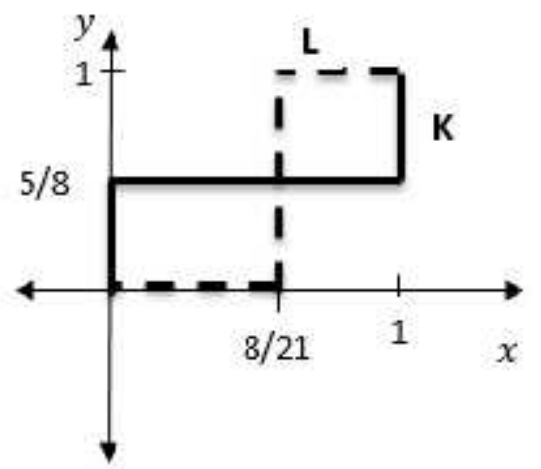

Figure 4

$$
\begin{array}{ccc}
\tilde{A} & =\left(\begin{array}{cc}
{[1,3]} & {[-2,12]} \\
{[-5,8]} & {[3,14]}
\end{array}\right) & \\
\tilde{p}=\tilde{a}_{11}+\tilde{a}_{22}-\tilde{a}_{21}-\tilde{a}_{12}=[-3,37] & \Rightarrow \ell(\tilde{p})=40, \ell(\tilde{q})=25 \\
\tilde{q}=\tilde{a}_{12}-\tilde{a}_{22}=[-9,16] & \Rightarrow \alpha=\frac{\ell(\tilde{q})}{\ell(\tilde{p})}=\frac{25}{40}=\frac{5}{8}>0
\end{array}
$$

Therefore,

$$
\left\{\begin{array}{rr}
(0, y), & y \leq \alpha=\frac{5}{8} \\
(1, y), & y \geq \frac{5}{8} \\
(x, y), & 0<x<1, y=\frac{5}{8}
\end{array}\right.
$$

Similarly, we obtain solutions set for player $I I$.

$$
\begin{array}{cc}
\tilde{B}=\left(\begin{array}{cc}
{[2,11]} & {[-1,3]} \\
{[2,5]} & {[1,6]}
\end{array}\right) & \Rightarrow \ell\left(\tilde{p}_{1}\right)=21, \ell\left(\tilde{q}_{1}\right)=8 \\
\tilde{p}_{1}=\tilde{b}_{11}+\tilde{b}_{22}-\tilde{b}_{21}-\tilde{b}_{12}=[-5,16] & \Rightarrow \beta=\frac{\ell\left(\tilde{q}_{1}\right)}{\ell\left(\tilde{p}_{1}\right)}=\frac{8}{21}>0
\end{array}
$$

the solutions for player $I I$ are obtained as follows,

$$
\left\{\begin{array}{cr}
(x, 0), & x \leq \beta=\frac{8}{21} \\
(x, 1), & x \geq \frac{8}{21} \\
(x, y), & 0<y<1, x=\frac{8}{21}
\end{array}\right.
$$

Hence, intersection point of $\mathrm{K}$ and $\mathrm{L}$ can be represented in the plain:

Hence, we have three intersection point in the plain $\left(x_{1}, y_{1}\right)=(0,0),\left(x_{2}, y_{2}\right)$ $=\left(\frac{8}{21}, \frac{5}{8}\right),\left(x_{3}, y_{3}\right)=(1,1)$.

For point $\left(x_{1}, y_{1}\right)=(0,0)$, the point $x_{1}=0 \quad\left(y_{1}=0\right)$ corresponds to mixed strategy $x_{1}^{*}=(0,1)\left(y_{1}^{*}=(0,1)\right)$ of player $I$ (player $\left.I I\right)$. Thus, $\left(x_{1}^{*}, y_{1}^{*}\right)$ is a pair of equilibrium of the game. For point $\left(x_{2}, y_{2}\right)=\left(\frac{8}{21}, \frac{5}{8}\right)$, the point $x_{2}=\frac{8}{21}$ $\left(y_{2}=\frac{5}{8}\right)$ corresponds to mixed strategy $x_{2}^{*}=\left(\frac{8}{21}, \frac{5}{8}\right)\left(y_{2}^{*}=\left(\frac{8}{21}, \frac{5}{8}\right)\right)$ of player $I$ 
(player $I I)$. Thus, $\left(x_{2}^{*}, y_{2}^{*}\right)$ is a pair of equilibrium of the game. Similarly, for point $\left(x_{3}, y_{3}\right)=(1,1)$, the point $x_{3}=1\left(y_{3}=1\right)$ corresponds to mixed strategy $x_{3}^{*}=(1,0)\left(y_{3}^{*}=(1,0)\right)$ of player $I$ (player $\left.I I\right)$. Thus, $\left(x_{3}^{*}, y_{3}^{*}\right)$ is a pair of equilibrium of the game. If players choose $\left(x_{1}^{*}, y_{1}^{*}\right)$ equilibrium strategy then the expected payoffs are as follows,

$$
\left(H_{I}\left(x_{1}^{*}, y_{1}^{*}\right), H_{I I}\left(x_{1}^{*}, y_{1}^{*}\right)\right)=([3,14],[1,6]) .
$$

If players choose $\left(x_{2}^{*}, y_{2}^{*}\right)$ equilibrium strategy then the expected payoffs are as follows,

$$
\left(H_{I}\left(x_{2}^{*}, y_{2}^{*}\right), H_{I I}\left(x_{2}^{*}, y_{2}^{*}\right)\right)=\left(\left[\frac{-217}{168}, \frac{737}{84}\right],\left[\frac{75}{56}, \frac{357}{56}\right]\right) .
$$

If players choose $\left(x_{3}^{*}, y_{3}^{*}\right)$ equilibrium strategy then the expected payoffs are as follows,

$$
\left(H_{I}\left(x_{3}^{*}, y_{3}^{*}\right), H_{I I}\left(x_{3}^{*}, y_{3}^{*}\right)\right)=([1,3],[2,11])
$$

\section{References}

[1] M. Sakawa, I. Nishizaki, Equilibrium solution in bimatrix games with fuzzy payoffs, Japanese Fuzzy Theory and Systems, 3 (1997), 307-324.

[2] J. Nash, Non-Cooperative Games, Annals of Mathematics, 54 (2),(1951), 286-295.

[3] G. Owen, Game Theory Third Edition Academic Press, (1995).

[4] R.E. Moore, Methods and Applications of Interval Analysis, SIAM, Philadelphia, USA, (1979).

[5] W.D. Collins, C. Hu, Studying interval valued matrix games with fuzzy logic Soft Computing, 12 (2), (2008), 147-155.

[6] W.D. Collins, C. Hu, Fuzzily determined interval matrix games, In: Proc. BISCSE 05, University of California, Berkeley (2005).

[7] P.K. Nayak, M. Pal, Bi-matrix games with interval payoffs and its Nash equilibrium strategy, Journal of Fuzzy Mathematics, 17(2), (2009), 421-435.

[8] A. Sengupta, T.K. Pal, D. Chakraborty, Interpretation of inequality constraints involving interval coefficients and a solution to interval linear programming, Fuzzy Sets and Systems, 119(1), (2001), 129-138.

[9] P.K. Nayak, M. Pal, Solution of rectangular interval games using graphical method, Tamsui Oxford Journal of Mathematical Sciences, 22 (1), (2006), 95-115.

[10] A. Sengupta, T.K. Pal, On comparing interval numbers, European Journal of Operational Research, 127, (2000), 28-43.

[11] H. Akyar, E.Akyar, A Graphical Method for Interval Matrix Games, Abstract and Applied Analysis, (2011), doi:10.1155/2011/260490. 